\title{
Engineered aminotransferase for the production of D-phenylalanine derivatives using biocatalytic cascades
}

\author{
Curtis J.W. Walton, ${ }^{[a]}$ Fabio Parmeggiani,,${ }^{[b]}$ Janet E.B. Barber, ${ }^{[a]}$ Jenna L. McCann, ${ }^{[a]}$ Nicholas J.
}

Turner, ${ }^{[b]}$ and Roberto A. Chica*[a]

\begin{abstract}
D-Phenylalanine derivatives are valuable chiral building blocks of a wide range of pharmaceuticals. Here, we developed stereoinversion and deracemization biocatalytic cascades allowing $\mathrm{D}$ phenylalanine derivatives containing electron-donating or withdrawing substituents of various sizes, and at different positions on the phenyl ring, to be synthesized with high enantiomeric excess $(90 \%$ to $>99 \%$ ee) from commercially-available racemic mixtures or L-amino acids. These whole-cell systems couple Proteus mirabilis L-amino acid deaminase with an engineered aminotransferase displaying nativelike activity towards D-phenylalanine, which we generated from Bacillus sp. YM-1 D-amino acid aminotransferase. Our cascades are applicable to preparative-scale synthesis and do not require cofactor regeneration systems or chemical reducing agents.
\end{abstract}

\section{Introduction}

D-Phenylalanine and its derivatives are essential building blocks in the synthesis of many pharmaceuticals, including antibiotics, ${ }^{[1]}$ antidiabetics, ${ }^{[2]}$ and chemotherapeutic agents for cancer treatment. ${ }^{[3]}$ Although asymmetric Strecker reactions can be used to synthesize these compounds, ${ }^{[4]}$ biocatalytic approaches are of great interest as they provide safer alternatives that exploit the unmatched selectivity of enzymes for the enantioselective synthesis of these chiral molecules. Previously, a fermentation process was developed to produce optically pure D-phenylalanine in E. coli cells. ${ }^{[5]}$ However, this process relied on an aromatic amino acid biosynthetic pathway specific to the phenylpyruvate precursor of D-phenylalanine, limiting its applicability to the synthesis of a wide range of substituted D-phenylalanine derivatives. More recently, we developed chemoenzymatic and biocatalytic cascades coupling L-amino acid deaminase (LAAD) with either phenylalanine ammonia-lyase ${ }^{[6]}$ or an engineered $D$ amino acid dehydrogenase ${ }^{[7]}$ to enable the production of optically pure D-phenylalanine derivatives. While these methods afford various D-phenylalanine derivatives with high enantiomeric excesses and conversion rates, they suffer from limitations such as low reactivity with substrates containing electron-donating substituents and requirement of a large excess of ammoniaborane reducing reagent, ${ }^{[6]}$ or the need for an additional cofactor

[a] C. J. W. Walton, J. E. B. Barber, J. L. McCann, Dr. R. A. Chica Department of Chemistry and Biomolecular Sciences, Centre for Catalysis Research and Innovation

University of Ottawa

10 Marie-Curie, Ottawa, Ontario, K1N 6N5, Canada

E-mail: rchica@uottawa.ca

[b] Dr. F. Parmeggiani, Dr. N. J. Turner

Manchester Institute of Biotechnology, School of Chemistry University of Manchester

131 Princess Street, M1 7DN Manchester, United Kingdom

Supporting information for this article is given via a link at the end of the document.

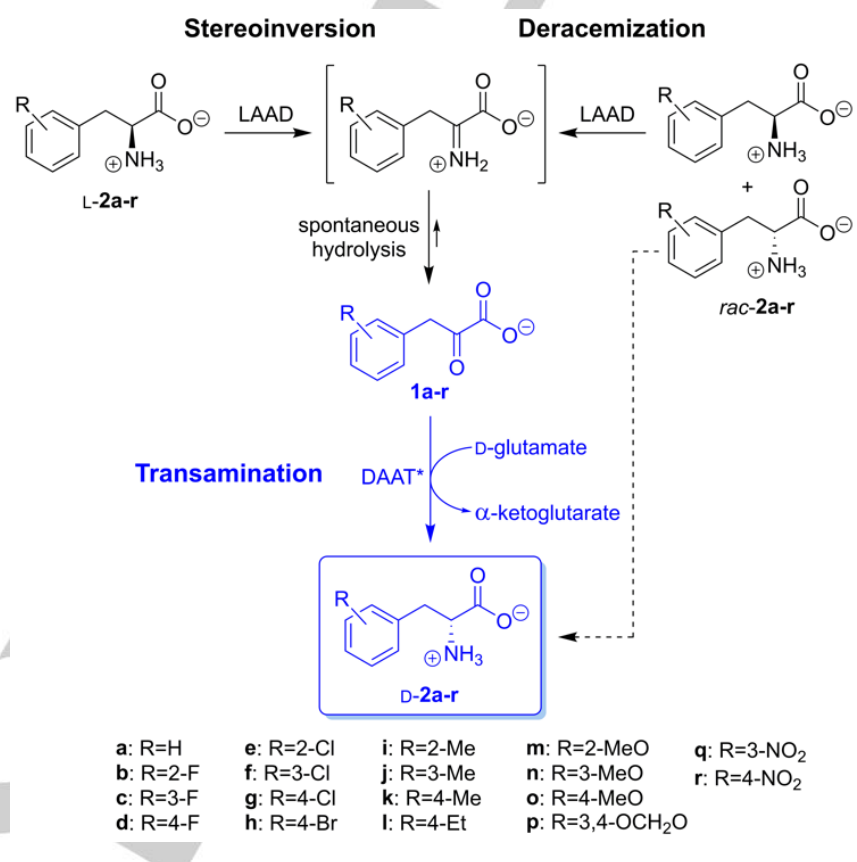

Scheme 1. Biocatalytic cascades for the asymmetric synthesis of phenylalanine derivatives. LAAD and DAAT* indicate L-amino acid deaminase and engineered $D$-amino acid aminotransferase, respectively.

recycling step. ${ }^{[7]}$ Attractive alternatives to these methods would be stereoinversion and deracemization cascades ${ }^{[8-12]}$ coupling LAAD with a D-phenylalanine-specific aminotransferase (Scheme 1). Such cascades would enable the one-pot enantioselective synthesis of a wide range of D-phenylalanine derivatives containing electron-donating or withdrawing substituents from more easily accessed L- or racemic phenylalanine derivatives, while having the further benefit of not requiring recycling of expensive cofactors. Although D-amino acid aminotransferases from various species have been shown to react with $D$ phenylalanine,$^{[13]}$ their activity towards this non-native substrate is low, ${ }^{[14-16]}$ motivating us to engineer more proficient biocatalysts to increase the efficiency and robustness of our targeted cascades.

\section{Results and Discussion}

As a starting point for biocatalyst engineering, we selected the D-amino acid aminotransferase (DAAT) from Bacillus sp. YM1. ${ }^{[17,18]}$ DAAT catalyzes the reversible transamination of $\mathrm{D}$-alanine and $\alpha$-ketoglutarate, yielding D-glutamate and pyruvate. DAAT can also react with the oxaloacetate acceptor substrate (Supplementary Results, Table S1) as well as with a range of polar and aliphatic D-amino acid donors, albeit with reduced specific activity. ${ }^{[15]}$ However, the specific activity of DAAT with D- 
phenylalanine is almost two orders of magnitude lower than with its native substrate D-alanine. ${ }^{[19]}$ We postulated that this lower activity with aromatic compounds results from the fact that the side chain of the D-amino acid substrate sits in a pocket formed by residues $\mathrm{V} 33, \mathrm{~S} 240$, and T242 in the DAAT active site ${ }^{[20]}$ (Figure 1) that is too small to efficiently bind the bulky benzyl side chain of D-phenylalanine. Previously, we demonstrated that replacement of $\mathrm{V} 33$ to a smaller glycine residue increased the catalytic efficiency $\left(k_{\text {cat }} / K_{\mathrm{M}}\right)$ of DAAT towards phenylpyruvate by

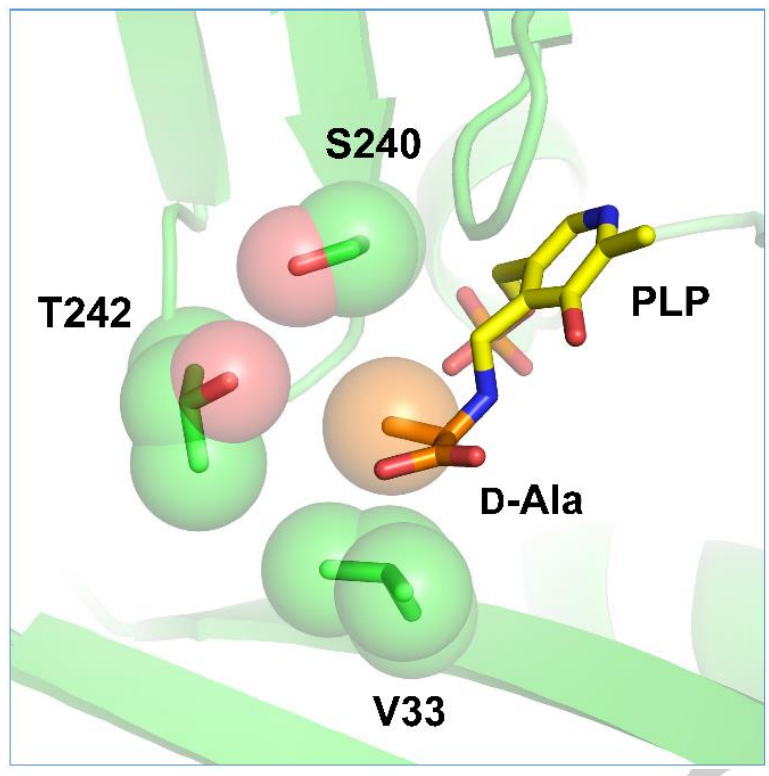

Figure 1. Active site of Bacillus sp. YM-1 DAAT. The side chain of the pyridoxal phosphate (PLP)-bound D-alanine donor substrate (orange) fits in a small cavity formed by residues V33, S240, and T242. PDB ID: 3DAA. ${ }^{[20]}$

over 3-fold, ${ }^{[21]}$ an enhancement that was mostly due to improved productive binding ( $K_{\mathrm{M}}$ was decreased by approximately 3 -fold). This result supports our hypothesis and suggests that mutations that increase the size of the side-chain binding pocket in the active site will lead to more efficient binding of bulkier substrates. While V33 is located on a $\beta$-strand, the two other residues forming the side-chain binding pocket (S240 and T242) are located on a loop formed by residues $240-243$. This structural element is expected to be more flexible than $\beta$-strands, leading us to hypothesize that substitution of these loop residues to glycine will permit a more efficient reshaping of the side-chain binding pocket for binding of bulkier substrates, and thus result in more efficient biocatalysts for the synthesis of D-phenylalanine derivatives.

To test our hypothesis, we prepared the S240G and T242G variants by site-directed mutagenesis and evaluated their ability to catalyze transamination with a library of D-amino acids, including several D-phenylalanine derivatives. To perform this analysis in a high-throughput manner, we developed a microplate-based colorimetric assay that utilizes bovine Daspartate oxidase (DDO) ${ }^{[2,23]}$ and horseradish peroxidase (HRP) as coupling enzymes (Scheme S1, Figures S1-S2, Table S1,
Supplementary Results). Using this assay, we measured the specific activity of wild-type and mutant DAATs towards a library of D-amino acids containing aliphatic, polar uncharged, basic, or aromatic side chains (Figure 2, Table S2). As expected, wild-type DAAT displays high specific activity with its native substrate Dalanine, reacts less efficiently with various aliphatic and polar substrates, and shows no detectable activity towards aromatic amino acids. Next, we screened the S240G and T242G mutants against the D-amino acid library, and included in this analysis the V33G variant that we previously described. ${ }^{[21]}$ We discovered that all three DAAT mutants display broader substrate specificity than the wild-type enzyme, with the V33G mutant having the broadest specificity as it reacts with all amino acids tested except for Dphenylglycine. Interestingly, none of the variants reacted with this aromatic amino acid, a result that can be rationalized by the absence of the methylene group bridging the main chain and phenyl ring in D-phenylglycine that is likely required to alleviate steric clashes of this ring with the loop formed by residues 240 243. All three mutants reacted with all D-phenylalanine derivatives tested with a specific activity $>10 \mathrm{mU} \mathrm{mg}^{-1}$, with the exception of S240G with D-2k. Of all the mutants, T242G had the highest specific activity with D-phenylalanine $\left(1210 \pm 60 \mathrm{mU} \mathrm{mg}^{-1}\right)$, a value that is within error to that of the wild type with its native substrate D-alanine $\left(1100 \pm 100 \mathrm{mU} \mathrm{mg}^{-1}\right)$. T242G is also highly active with all D-phenylalanine derivatives tested, suggesting that we successfully engineered an efficient aminotransferase biocatalyst for the synthesis of D-phenylalanine and its derivatives.

D-Amino acid
WT

$\leq 10 \mathrm{mU} \mathrm{mg}^{-1} \quad 10 \mathrm{mU} \mathrm{mg}^{-1}<\odot<100 \mathrm{mU} \mathrm{mg}^{-1} \quad \geq 100 \mathrm{mU} \mathrm{mg}^{-1}$

Figure 2. Substrate specificity profiles of wild-type and mutant DAAT enzymes Each dot represents a unique aminotransferase/amino acid combination and is colored based on the specific activity of the resulting enzymatic reaction. The acceptor substrate for each reaction was oxaloacetate.

To gain a better understanding of the underlying reason for the increased activity of these mutants towards D-phenylalanine 
Table 1. Apparent kinetic parameters for the transamination of various D-phenylalanine derivatives by wild-type and mutant DAATs.

\begin{tabular}{|c|c|c|c|c|c|c|}
\hline Enzyme & Substrate ${ }^{[a]}$ & $\mathbf{R}$ & $\begin{array}{c}K_{\mathrm{M}} \\
(\mathrm{mM})\end{array}$ & $\begin{array}{l}k_{\text {cat }} \\
\left(\mathbf{s}^{-1}\right)\end{array}$ & $\begin{array}{c}K_{\mathrm{cat}} / K_{\mathrm{M}} \\
\left(\mathrm{M}^{-1} \mathbf{s}^{-1}\right)\end{array}$ & $\begin{array}{c}\text { Relative } \\
\boldsymbol{k}_{\text {cat }} / K_{\mathrm{M}}\end{array}$ \\
\hline \multirow[t]{5}{*}{ Wild type } & $\mathrm{D}-2 \mathrm{a}$ & $\mathrm{H}$ & N.D..$^{[b]}$ & N.D..$^{[b]}$ & 0.65 & 1 \\
\hline & $D-2 j$ & 3-Me & N.D..$^{[b]}$ & N.D..$^{[b]}$ & 1.5 & 1 \\
\hline & $D-2 n$ & 3-MeO & $3.7 \pm 0.4$ & $0.038 \pm 0.001$ & 10 & 1 \\
\hline & $\mathrm{D}-2 \mathrm{q}$ & $3-\mathrm{NO}_{2}$ & $2.4 \pm 0.3$ & $0.025 \pm 0.001$ & 10 & 1 \\
\hline & $\mathrm{D}-2 \mathrm{k}$ & 4-Me & $1.4 \pm 0.5$ & $0.006 \pm 0.001$ & 4 & 1 \\
\hline \multirow[t]{5}{*}{ V33G } & $\mathrm{D}-2 \mathrm{a}$ & $\mathrm{H}$ & $8.8 \pm 0.4$ & $0.27 \pm 0.01$ & 31 & 48 \\
\hline & $\mathrm{D}-\mathbf{2} \mathbf{i}$ & 2-Me & $18 \pm 2$ & $0.59 \pm 0.04$ & 33 & $>3300$ \\
\hline & $\mathrm{D}-\mathbf{2 j}$ & 3-Me & $17.8 \pm 0.8$ & $2.60 \pm 0.05$ & 146 & 97 \\
\hline & $D-2 n$ & 3-MeO & $8.9 \pm 0.9$ & $0.43 \pm 0.02$ & 48 & 5 \\
\hline & $D-2 q$ & $3-\mathrm{NO}_{2}$ & $8.5 \pm 0.8$ & $1.44 \pm 0.06$ & 169 & 17 \\
\hline \multirow[t]{6}{*}{ S240G } & $\mathrm{D}-2 \mathrm{a}$ & $\mathrm{H}$ & $15 \pm 2$ & $0.58 \pm 0.04$ & 39 & 60 \\
\hline & $\mathrm{D}-2 \mathbf{i}$ & 2-Me & $>50$ & $<0.0006$ & $<0.01$ & 1 \\
\hline & $D-2 j$ & 3-Me & $16 \pm 2$ & $1.6 \pm 0.1$ & 100 & 67 \\
\hline & $D-2 n$ & 3-MeO & $4.0 \pm 0.8$ & $0.26 \pm 0.02$ & 65 & 6.5 \\
\hline & $D-2 q$ & $3-\mathrm{NO}_{2}$ & $3.0 \pm 0.5$ & $0.30 \pm 0.02$ & 100 & 10 \\
\hline & $\mathrm{D}-2 \mathrm{k}$ & 4-Me & $26 \pm 5$ & $0.8 \pm 0.1$ & 31 & 8 \\
\hline \multirow[t]{6}{*}{ T242G } & $\mathrm{D}-2 \mathrm{a}$ & $\mathrm{H}$ & $4.5 \pm 0.8$ & $1.8 \pm 0.1$ & 400 & 615 \\
\hline & $D-2 \mathbf{i}$ & 2-Me & $16 \pm 1$ & $0.74 \pm 0.03$ & 45 & $>4500$ \\
\hline & $\mathrm{D}-2 \mathbf{j}$ & 3-Me & $3.7 \pm 0.7$ & $2.6 \pm 0.1$ & 703 & 468 \\
\hline & $D-2 n$ & 3-MeO & $6 \pm 2$ & $2.2 \pm 0.4$ & 367 & 37 \\
\hline & $D-2 q$ & $3-\mathrm{NO}_{2}$ & $5.3 \pm 0.7$ & $0.44 \pm 0.01$ & 83 & 8 \\
\hline & $\mathrm{D}-2 \mathrm{k}$ & 4-Me & $5 \pm 1$ & $2.2 \pm 0.2$ & 449 & 112 \\
\hline
\end{tabular}

[a] For all reactions, the acceptor substrate was $5 \mathrm{mM}$ oxaloacetate (mean \pm s.d., $\mathrm{n}=3$ ). [b] N.D.: could not be determined as substrate saturation was not possible within its solubility limit.

derivatives, we determined steady-state kinetic parameters of wild-type and mutant DAATs for transamination of six representative substrates containing either electron-donating or withdrawing substituents at the ortho, meta, or para positions of the phenyl ring (Table 1). For wild-type DAAT, we were unable to achieve saturation with D-phenylalanine within its solubility limit, which prevented reliable determination of $K_{\mathrm{M}}$ and $k_{\text {cat }}$. However, we were able to measure a catalytic efficiency of $0.65 \mathrm{M}^{-1} \mathrm{~s}^{-1}$ for this substrate, a value that is over three orders of magnitude lower than for the native substrate D-alanine (Table S1). All other Dphenylalanine derivatives tested with wild-type DAAT also gave low catalytic efficiencies $\left(\leq 10 \mathrm{M}^{-1} \mathrm{~s}^{-1}\right)$, confirming that wild-type DAAT is a poor biocatalyst for transamination of aromatic D-amino acids. On the other hand, all three DAAT mutants displayed enhanced catalytic efficiency towards aromatic D-amino acids compared to the wild type, as observed during screening. Surprisingly, the higher catalytic efficiencies result mostly from improved $k_{\text {cat }}$ and not $K_{\mathrm{M}}$, suggesting that these mutations enhance catalytic activity without significantly improving productive binding, contrary to our hypothesis. In agreement with our screening results, the T242G mutant had the highest catalytic efficiency for D-phenylalanine $\left(400 \mathrm{M}^{-1} \mathrm{~s}^{-1}\right)$, which represents a 615 -fold increase relative to the wild type. Importantly, this $K_{\text {cat }} / K_{M}$ value is only 4.6-fold lower than that of the wild type with its native substrate D-alanine (Table S1), and the $k_{\text {cat }}$ of the mutant (1.8 \pm $\left.0.1 \mathrm{~s}^{-1}\right)$ is 1.5 -fold greater than that of the wild type with its native substrate $\left(1.18 \pm 0.03 \mathrm{~s}^{-1}\right)$. Taken together, these results confirm that we have successfully engineered an aminotransferase with native-like catalytic activity towards D-phenylalanine and its derivatives.

Next, we performed a series of transamination reactions with our most active mutant, DAAT-T242G, to synthesize D-phenylalanine derivatives. An E. coli whole-cell biocatalyst expressing DAATT242G was incubated with a panel of phenylpyruvate analogs containing electron-donating or withdrawing substituents of different sizes and at different positions on the phenyl ring as acceptor substrates, along with the D-glutamate donor. All phenylpyruvate analogs tested were transformed into the corresponding D-phenylalanine derivatives with high conversion rates $(\geq 94 \%$ ) over a 12 -hour period (Table 2$)$, confirming the broad specificity of our biocatalyst. The enantiomeric excesses of 


\begin{tabular}{|c|c|c|c|}
\hline Substrate & $\mathbf{R}$ & Conv. (\%) ${ }^{[a]}$ & ee $\mathrm{D}-2(\%)^{[b]}$ \\
\hline \multicolumn{4}{|c|}{ Transamination $[\mathrm{c]}$} \\
\hline $1 \mathrm{a}$ & $\mathrm{H}$ & $>99$ & $95(99)$ \\
\hline $1 d$ & $4-\mathrm{F}$ & $>99$ & $92(>99)$ \\
\hline $1 e$ & $2-\mathrm{Cl}$ & $>99$ & $92(>99)$ \\
\hline 1h & $4-\mathrm{Br}$ & 98 & $80(98)$ \\
\hline $1 \mathrm{i}$ & $2-M e$ & 94 & 99 (>99) \\
\hline $1 \mathrm{k}$ & 4-Me & 98 & $93(98)$ \\
\hline 11 & $4-E t$ & $>99$ & $97(>99)$ \\
\hline $1 \mathrm{~m}$ & $2-\mathrm{MeO}$ & 97 & $98(98)$ \\
\hline 10 & $4-\mathrm{MeO}$ & $>99$ & $99(>99)$ \\
\hline $1 p$ & $3,4-\mathrm{OCH}_{2} \mathrm{O}$ & 98 & 96 (>99) \\
\hline \multicolumn{4}{|c|}{ Stereoinversion/Deracemization $[\mathrm{d]}]$} \\
\hline rac-2a & $\mathrm{H}$ & - & $>99(94)$ \\
\hline rac-2b & $2-\mathrm{F}$ & - & $>99$ (98) \\
\hline rac-2c & $3-\mathrm{F}$ & - & $99(97)$ \\
\hline rac-2d & $4-\mathrm{F}$ & - & $99(97)$ \\
\hline L-2e & $2-\mathrm{Cl}$ & - & $98(83)$ \\
\hline L-2f & $3-\mathrm{Cl}$ & - & 99 (89) \\
\hline rac-2g & $4-\mathrm{Cl}$ & - & $>99$ (97) \\
\hline rac-2h & $4-\mathrm{Br}$ & - & $>99$ (>99) \\
\hline L-2i & 2-Me & - & $90(86)$ \\
\hline L-2j & 3-Me & - & $96(76)$ \\
\hline L-2k & 4-Me & - & $93(79)$ \\
\hline L-2n & 3-MeO & 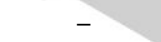 & $98(83)$ \\
\hline
\end{tabular}

[a] Conversion of $\mathbf{1}$ to $\mathbf{2}$ measured by reverse-phase HPLC on a nonchiral stationary phase. [b] For transamination, the values given are after $12 \mathrm{~h}$ incubation with whole-cell biocatalyst (values in parenthesis are with purified enzyme) whereas for stereoinversion/deracemization, the values are after $4 \mathrm{~h}$ (values in parenthesis are after $2 \mathrm{~h}$ ). [c] Reaction conditions: $5 \mathrm{mM} \mathrm{1,} 20 \mathrm{mM}$ D-Glu, $0.5 \mathrm{mM} \mathrm{PLP}, 1 \% \mathrm{v} / \mathrm{v}$ DMSO, $20 \mathrm{mg} \mathrm{mL}^{-1}$ DAAT-T242G wet cells (or $1 \mathrm{mg} \mathrm{mL}^{-1}$ purified enzyme), $100 \mathrm{mM}$ potassium phosphate buffer, $\mathrm{pH} 8.0,37^{\circ} \mathrm{C}, 12 \mathrm{~h}$. [d] Reaction conditions: $10 \mathrm{mM} \mathrm{2,30} \mathrm{mM} \mathrm{D-Glu,} 20 \mathrm{mg} \mathrm{mL}^{-1}$ LAAD wet cells, $20 \mathrm{mg} \mathrm{mL}^{-1}$ DAAT-T242G wet cells, $100 \mathrm{mM}$ potassium phosphate buffer, $\mathrm{pH} 8.0,37^{\circ} \mathrm{C}, 4 \mathrm{~h}$

these transamination reactions were also high (92-99\%), with the exception of the transamination of $\mathbf{1 h}$, which resulted in an ee value of $80 \%$. It is unclear what caused the lower enantiomeric excess obtained with this substrate as the enzyme displays nearperfect enantioselectivity (e.g., ee $\geq 98 \%$ for all transamination reactions using purified enzyme, Table 2), but it may result from unrelated enzymatic processes that occur in $E$. coli cells over the 12-hour incubation.

Having confirmed that our DAAT-T242G whole-cell biocatalyst could be used to synthesize a variety of D-phenylalanine derivatives from their corresponding keto acids, we combined it with an $E$. coli whole-cell biocatalyst expressing LAAD from Proteus mirabilis ${ }^{[24]}$ to test our targeted stereoinversion and deracemization cascades (Scheme 1). These cascades should enable the one-pot tandem oxidative deamination and reductive amination of L-amino acids into D-amino acids. Starting from racemic or L-phenylalanine derivatives containing electronwithdrawing (2b-h) or donating (2i-k, n) substituents at the ortho, meta, or para positions of the phenyl ring, our biocatalytic cascades yielded the corresponding D-phenylalanine derivatives with excellent enantiomeric excesses $(90 \%$ to $>99 \%)$ in 4 hours (Table 2). Even in the case where transamination of the corresponding phenylpyruvate does not proceed with high enantioselectivity (e.g., 1h), very high ee can be obtained because repeated deracemization cycles ultimately result in complete consumption of the L-enantiomer and accumulation of enantiopure D-product. Furthermore, no accumulation of the corresponding phenylpyruvate was observed during deracemization (Figure S3), strongly suggesting that the undesired L-enantiomer was converted to the D-enantiomer and not simply oxidized by LAAD. Thus, our biocatalytic processes are true stereoinversion and deracemization cascades and not kinetic resolution processes.

To confirm this result and demonstrate the practical utility of our biocatalytic cascades for the synthesis of valuable Dphenylalanine derivatives, we performed a preparative scale test of the deracemization cascade with rac-2d and the D-glutamate donor to produce D-4-fluorophenylalanine, a building block of melanocortin-4 receptor agonists used as antiobesity agents. ${ }^{[25]}$ To favor complete conversion of racemic $\mathbf{2 d}$ into enantiopure D2d, an excess of D-glutamate was used (3 equiv.). Although complete deracemization was observed (ee $=99 \%$ ), the unreacted excess D-glutamate could not be separated from D-2d by adsorption on ion-exchange resin, our standard procedure for amino acid purification (Figure S4a). To address this issue, we envisaged to incubate the reaction mixture with DDO following completion of the deracemization process, in order to transform unreacted donor substrate into its corresponding keto acid, which can be readily removed by ion-exchange purification. We tested the addition of wet $E$. coli cells expressing DDO (20 or $80 \mathrm{mg} \mathrm{mL}$ ${ }^{1}$ for 24 or $8 \mathrm{~h}$, respectively) to deracemization mixtures prepared using excess D-glutamate or D-aspartate. D-Aspartate was included in our tests because it is the preferred substrate of DDO (Figure S1a) and also a good donor substrate of DAAT. ${ }^{[26]}$ DDO treatment enabled the complete removal of excess D-aspartate but not D-glutamate after deracemization of rac-2d under these conditions (Figure S4), a result that can be explained by the 8 -fold higher catalytic efficiency of DDO towards D-aspartate (Table S1). Using this procedure with the D-aspartate donor, we were able to obtain $76.9 \mathrm{mg}$ of optically pure D-2d (99\% ee), representing an 
isolated yield of $84 \%$. These results confirm that our deracemization cascade can be used for the preparative scale synthesis of valuable enantiopure D-phenylalanine derivatives with high yield.

\section{Conclusions}

We have successfully developed one-pot biocatalytic processes for the synthesis of valuable enantiopure D-phenylalanine derivatives containing electron-donating or withdrawing substituents of various sizes, and at different positions on the phenyl ring, from more accessible starting materials. To do this, we engineered an aminotransferase displaying high catalytic activity towards D-phenylalanine. In comparison with previous biocatalytic cascades used to synthesize similar compounds, our cascades have the benefits of not requiring expensive cofactors, ${ }^{[7]}$ removing the need for a cofactor regeneration system, or large excesses of chemical reducing agents. ${ }^{[6]}$ However, our cascades require stoichiometric amounts of the D-glutamate or D-aspartate donor substrate, which may represent a disadvantage for certain applications. This disadvantage can be eliminated by generating the donor substrate in situ with a glutamate or aspartate racemase, ${ }^{[27,28]}$ and by replacing LAAD from Proteus mirabilis with that from Proteus myxofaciens, which has been shown to not deaminate L-glutamate or L-aspartate. ${ }^{[29,30]}$ Although this study focused on the synthesis of D-phenylalanine derivatives, other Damino acids could also be synthesized using our cascades, since all three DAAT variants reported here display increased activity relative to the wild type towards a series of D-amino acids displaying aliphatic or polar side chains. Our cascades thus complement other previously reported biocatalytic processes, ${ }^{5-}$ 7,31] and represent useful additions to the D-amino acid synthesis toolbox.

\section{Experimental Section}

\section{Experimental details are provided in the supplementary materials.}

\section{Acknowledgements}

R.A.C. acknowledges an Early Researcher Award from the Ontario Ministry of Economic Development \& Innovation (ER1410-139), and grants from the Natural Sciences and Engineering Research Council of Canada (RGPIN-2016-04831) and the Canada Foundation for Innovation (26503). F.P. was supported by the Biotechnology and Biological Sciences Research Council (BBSRC) and Glaxo-SmithKline (GSK) under the Strategic Longer and Larger (sLoLa) grant initiative ref. BB/K00199X/1. N.J.T. thanks the Royal Society for a Wolfson Research Merit Award.

Keywords: biocatalysis • enzyme engineering • transaminases • D-amino acids $\bullet$ D-aspartate oxidase $\cdot$ rational design
[1] K. Miyao, Bull. Agric. Chem. Soc. Jpn. 1960, 24, 23-30.

[2] N. Tentolouris, C. Voulgari, N. Katsilambros, Vasc Heal. Risk Manag 2007, 3, 797-807.

[3] M. Kovacs, A. V. Schally, B. Csernus, Z. Rekasi, Proc. Natl. Acad. Sci. U. S. A. 2001, 98, 1829-34.

[4] J. Wang, X. Liu, X. Feng, Chem. Rev. 2011, 111, 69476983.

[5] I. G. Fotheringham, P. P. Taylor, J. L. Ton (Monsanto), US 5728555 A, 1998.

[6] F. Parmeggiani, S. L. Lovelock, N. J. Weise, S. T. Ahmed N. J. Turner, Angew. Chemie - Int. Ed. 2015, 54, 4608-4611.

[7] F. Parmeggiani, S. T. Ahmed, M. P. Thompson, N. J. Weise,

J. L. Galman, D. Gahloth, M. S. Dunstan, D. Leys, N. J. Turner, Adv. Synth. Catal. 2016, 358, 3298-3306.

[8] J. H. Schrittwieser, S. Velikogne, M. Hall, W. Kroutil, Chem. Rev. 2017, DOI 10.1021/acs.chemrev.7b00033.

[9] S. P. France, L. J. Hepworth, N. J. Turner, S. L. Flitsch, ACS Catal. 2017, 7, 710-724

[10] P. A. Santacoloma, G. Sin, K. V. Gernaey, J. M. Woodley, Org. Process Res. Dev. 2010, 15, 203-212.

[11] E. Ricca, B. Brucher, J. H. Schrittwieser, Adv. Synth. Catal. 2011, 353, 2239-2262.

[12] N. Oberleitner et al., ChemCatChem 2013, 5, 3524-3528 see supporting information.

[13] R. X. Liu, S. P. Liu, S. Cheng, L. Zhang, Z. Y. Ding, Z. H. Gu, G. Y. Shi, Appl. Biochem. Microbiol. 2015, 51, 695-703.

[14] K. Yonaha, H. Misono, T. Yamamoto, K. Soda, J. Biol. Chem. 1975, 250, 17, 6983-6989.

[15] J. Kobayashi, Y. Shimizu, Y. Mutaguchi, K. Doi, T. Ohshima, J. Mol. Catal. B Enzym. 2013, 94, 15-22.

[16] F. Khorsand, C. D. Murphy, A. J. Whitehead, P. C. Engel, Green Chem. 2017, 19, 503-510.

[17] K. Tanizawa, S. Asano, Y. Masu, S. Kuramitsu, H. Kagamiyama, H. Tanaka, K. Soda, J. Biol. Chem. 1989, 264, 2450-2454.

[18] K. Tanizawa, Y. Masus, S. Asano, H. Tanaka, K. Sodas, J. Biol. Chem. 1989, 264, 2445-2449.

[19] Y. Fuchikami, T. Yoshimura, A. Gutierrez, N. Esaki, J. Biochem. 1998, 910, 905-910.

[20] D. Peisach, D. M. Chipman, P. W. Van Ophem, J. M. Manning, D. Ringe, Biochemistry 1998, 37, 4958-4967.

[21] J. E. B. Barber, A. M. Damry, G. F. Calderini, C. J. W. Walton, R. A. Chica, Anal. Biochem. 2014, 463, 23-30.

[22] T. Simonic, D. Stefano, A. Negri, G. Tedeschi, M. Malcovati, M. L. Tenchini, S. Ronchi, Biochem. J. 1997, 322, 729-735.

[23] A. Negri, G. Tedeschi, F. Ceciliani, S. Ronchi, Biochim. Biophys. Acta, Protein Struct. Mol. Enzymol. 1999, 1431, 212222.

[24] G. Massad, H. Zhao, H. L. Mobley, J. Bacteriol. 1995, 177, 5878-5883.

[25] X. Tian et al., J. Med. Chem. 2008, 51, 6055-6066 see supporting information.

[26] A. Gutierrez, T. Yoshimura, Y. Fuchikami, K. Soda, N. Esaki, Protein Eng. 1998, 11, 53-8.

[27] J. Ahn, J. H. Chang, K. Kim, FEBS Lett. 2015, 589, 38423847.

[28] T. Washio, S. Kato, T. Oikawa, Extremophiles 2016, 20, 711-721.

[29] P. Motta, G. Molla, L. Pollegioni, M. Nardini, J. Biol. Chem. 2016, 291, 10457-10475.

[30] P. P. Taylor, D. P. Pantaleone, R. F. Senkpeil, I. G. Fotheringham, Trends Biotechnol. 1998, 16, 412-418.

[31] A. S. Bommarius, M. Schwarm, K. Drauz, Chima 2001, 55, 50-59. 
WILEY-VCH

FULL PAPER 


\section{Entry for the Table of Contents}

\section{FULL PAPER}

D-Phenylalanine derivatives are valuable chiral building blocks of many pharmaceuticals. The development of an E. coli whole-cell system coupling Proteus mirabilis L-amino acid deaminase with an engineered aminotransferase displaying nativelike activity towards D-phenylalanine allows for the synthesis of these derivatives with high enantiomeric excess from commercially-available racemic mixtures or L-amino acids.

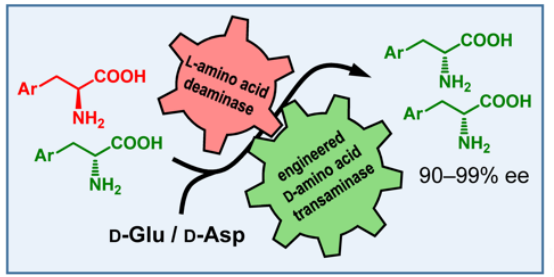

Curtis J.W. Walton, Fabio Parmeggiani, Janet E.B. Barber, Jenna L. McCann, Nicholas J. Turner, Roberto A. Chica*

Page No. - Page No.

Engineered aminotransferases for the production of $\mathrm{D}$-phenylalanine derivatives using biocatalytic cascades. 